\title{
Cytogenetics of human malignant melanoma
}

Jeffrey M. Trent

Emanuel N. Maisel Professor of Oncology, Department of Radiation Oncology and

Human Genetics, 1150 W. Medical Center Drive, University of Michigan, Ann Arbor, MI 48109-0668, USA

Key words: cytogenetics, malignant melanoma

\begin{abstract}
There has been a tremendous recent resurgence of interest in examining chromosomal abnormalities in human cancers (particularly solid tumors). This interest has been stimulated by the molecular examination of recurring chromosome abnormalities, and the recognition that they may pinpoint the location of growth regulatory sequences (e.g. cellular oncogenes). This finding coupled with the clear recognition that specific chromosome abnormalities can also have important diagnostic and prognostic implications, have caused this avenue of research to expand at a significant rate. The following brief review will summarize the current state of knowledge regarding recurring chromosome abnormalities in human malignant melanoma. A discussion of chromosome changes in pre-malignant skin lesions, primary melanoma, and metastatic melanoma is described. Brief descriptions of the potential clinical utility, and biologic relevance of chromosome abnormalities in this disorder are also discussed.
\end{abstract}

\section{Introduction}

Based upon epidemiologic grounds there has been a significant increase in the incidence of malignant melanoma worldwide [1]. In North America a doubling of the incidence rate for melanoma has occurred each decade for the past forty years, and the current estimation is that $>1 / 100$ Americans will develop melanoma by the year 2000 [1]. These alarming statistics coupled with the realization that three fourths of all deaths from skin cancer result from melanoma strongly suggests that significant attention should be focused on understanding not just the clinical behavior, but also the biology and genetics of this disorder. In this brief review, emphasis will be exclusively placed upon a discussion of the current state of knowledge of chromosomal alterations in this important neoplasm. However, the underlying viewpoint is that a proportion of these chromosomal changes will in fact represent byproducts of molecular events which themselves may play a causative role in melanoma carcinogenesis.

\section{General overview}

Cytogenetic examination of human cancers has significantly expanded over the past decade with more than 14,000 cases now reported in the world literature [2]. However, our knowledge base is weighted heavily toward human hematopoetic malignancies, with fewer than $20 \%$ of studies focusing on human solid tumors (which represent $80 \%$ of the cancers of man) [2,3]. Nevertheless, the identification of recurring sites of chromosome change in human solid tumors (including melanoma) is increasing at a tremendous rate. The following sections will briefly discuss chromosomal alterations in malignant melanoma. A brief discussion of chromosome alterations in premalignant nevi and early stage melanomas will be presented first, followed by a 
discussion of chromosomal changes occurring in metastatic disease. Finally discussion of very preliminary work suggesting that chromosomal abnormalities may correlate with clinical outcome in malignant melanoma, as well as studies related to the biologic significance of chromosome abnormalities will be presented.

\section{Cytogenetic analysis of premalignant nevi and primary melanoma}

The recognition of recurring sites of chromosome change has pinpointed several genes involved in cellular transformation. Accordingly, an examination of the earliest stages of tumor development, or the study of premalignant precursor lesions may provide significant information of biologic relevance. Unfortunately, there have been few reports on the cytogenetics of early malanocytic lesions, either common nevi, dysplastic nevi, or early (radial growth phase) primary melanomas. The studies undertaken to date have focused on two different approaches, first analyzing the constitutional karyotypes of patients with dysplastic nevus syndrome (DNS) [4, 5], or familial atypical multiplemole melanoma (FAMMM) [6]. Second, cytogenetic examination of melanocytes from dysplastic nevi and primary melanoma.

Cytogenetic studies of peripheral blood lymphocytes from DNS or FAMMM patients have been undertaken because both represent inherited disorders characterized by the occurrence of multiple atypical moles and a strongly increased risk for melanoma. The mode of inheritance appears to be autosomal dominate for both disorders, although there is a considerable degree of non-penetrance. Chromosome analyses on both DNS and FAMMM patient populations have suggested either normal karotypes with normal spontaneous sister chromatid exchange rates in fibroblasts from these individuals, or slightly enhanced numbers of nonclonal cytogenetic abnormalities in affected versus control patients $[4,5,7]$. Specifically, some studies have shown a statistically significant increase in major structural abnormalities in the peripheral blood lymphocytes of melanoma patients, while numeric abnormalities (again non-clonal and random) are observed more frequently in patients with DNS. Unfortunately, because no clonal cytogenetic abnormalities have yet been observed in any family with a hereditary disorder predisposing the melanoma, the results of generalized chromosome instability are difficult to interpret. Nevertheless, there does appear to be some slight increase in the level of spontaneous structural and numeric chromosome abnormalities in patients predisposed to the development of malignant melanoma.

In contrast to the cytogenetic study of peripheral blood from a reasonably large number of melanoma prone families, relatively few studies have focused on cytogenetic analysis of melanocytes from premalignant nevi and primary melanomas [8-10]. To date the concensus appears to be that chromosome abnormalities do exist in early stages of melanocytic neoplasia, and of interest the results are surprisingly non-overlapping between abnormalities observed in later stage melanomas. Parmiter et al. [9] studied a series of melanotic tumors including common nevi through metastatic melanomas and their data supported the view that terminal region of chromosome $10 \mathrm{q}$ (bands $\mathrm{q} 24-\mathrm{q} 26$ ) may harbor one or more genes involved in the early stages of melanocytic neoplasia. In contrast, Cowan et al. [8] analyzed cultures from a variety of lesions, including congenital and dysplastic nevi, primary melanoma and metastatic melanoma with results indicating that chromosome 9 (especially band region 9pter-p22) may be the site of a gene(s) important in the early steps of malignant transformation of melanocytes. In the study of Cowan [8], 22/26 cultures derived from dysplastic nevi had totally normal karyotypes, while $100 \%$ of cultures from primary and metastatic melanomas showed chromosome abnormalities. Chromosome 9 as well as chromosome 11 were the only two chromosomes altered in common between nevi and abnormal karyotypes of the malignant melanomas examined. In another study by McDonagh et al. [10], a patient with an 11p-constitutional abnormality was reported who developed dysplastic nevi at an early age.

These limited but provocative studies make up a selected, but representative base of knowledge regarding cytogenetic analysis from premalignant 


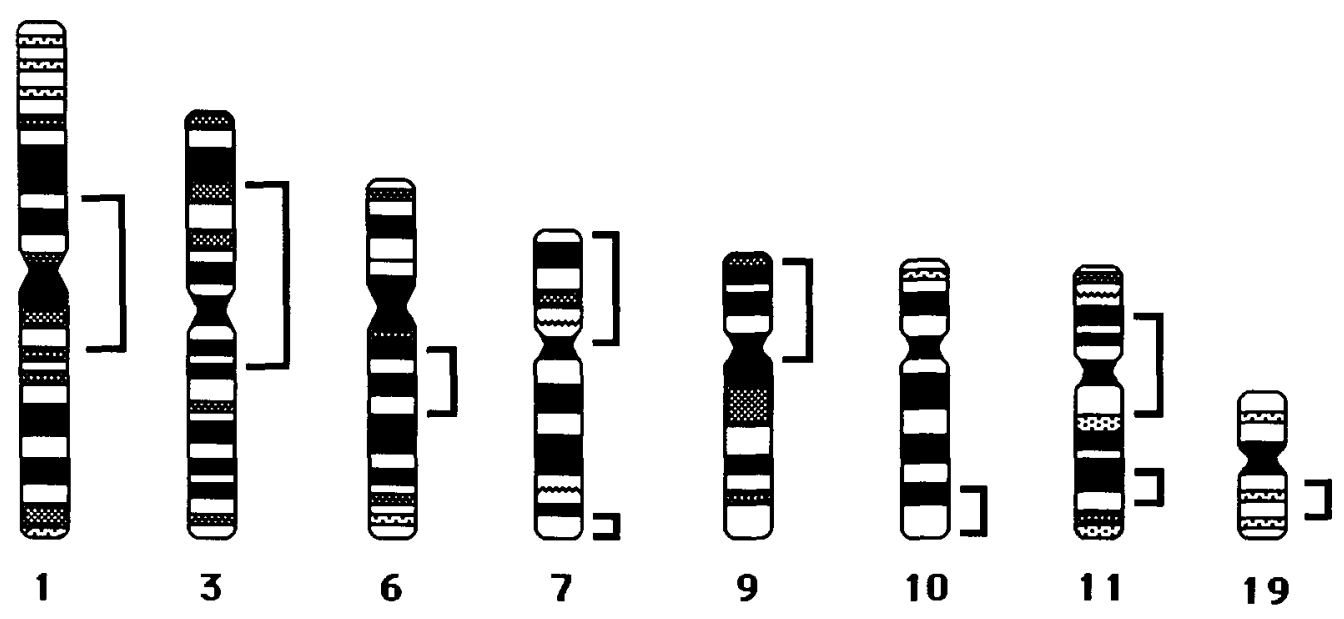

Fig. 1.Ideograms of human chromosomes most frequently altered in human malignant melanoma. The brackets indicate the most frequently altered band regions.

melanomas. To summarize, results to date suggest that patients with a hereditary predisposition for melanoma formation may show slightly increased levels of spontaneous chromosome abnormalities in both peripheral blood lymphocytes and human skin fibroblasts. Although, direct cytogenetic examination of dysplastic nevi and primary melanomas are still based on very small patient numbers, the results clearly suggest that chromosomes $9 q$ and $10 \mathrm{q}$ may be important sites of chromosome change involved early in the genesis of this disorder. The gene(s) which may be disregulated by these chromosome abnormalities are not identified. However Cowan et al. [8] has suggested that the interferon gene cluster which maps to chromosome $9 p$ [11] may be a possible target for the $9 q$ chromosome rearrangements.

\section{Chromosome abnormalities in metastatic malignant melanoma}

A number of studies have appeared characterizing chromosomal alterations in metastatic melanoma [12-24]. Unfortunately, several variables exist in regards to the cultured cells so many of these reports have involved studies of established melanoma cell lines, and even studies of primary outgrowths have included a number of variables in technical procedures (e.g. days in culture, culture media, chromosome harvesting techniques, etc.). However, despite these variables it is surprising that a general pattern of cytogenetic alteration in metastatic melanoma is beginning to emerge. First, $\geq 95 \%$ of melanomas studied from primary outgrowths have recognizable chromosomal abnormalities, and the modal chromosome number is consistently in the near-triploid range. For example, a recent study from this laboratory [19], the modal chromosome number of 27 patients was 61 chromosomes/cell, with modal numbers ranging from $\sim 40-90$ chromosomes/cell.

Within this framework of multiple chromosomal rearrangements a number of chromosomes appear to be non-randomly involved in structural rearrangements including: $1,3,6,7,9,10,11$ and 19 .

Table 1 provides a listing of those recurring chromosomal alterations currently recognized in malignant melanoma by the Committee on Structural Chromosomal Changes in Neoplasia of the Human Gene Mapping (HMG) Workshop [3]. This listing recognizes only those abnormalities which have been reported by more than one laboratory, and in sufficient numbers to meet the criteria for HMG10.5 [3]. In addition to these recognizable changes melanomas are often characterized by the presence of unidentifiable markers (umars), which are a feature of many solid tumors and occur in $\sim 60 \%$ of metastatic melanomas studied.

One feature of the chromosome abnormalities of 
melanoma which is common to other solid tumors is the finding that chromosome alterations often characterize a chromosome band region, rather than a specific unique translocation (e.g. the $\mathrm{t}(9 ; 22)$ translocation characterizing chronic myelogenous leukemia). Accordingly, as illustrated in Table 1, deletions of varying size (or translocations with various autosomal partners) may pinpoint a 'region' most frequently altered, rather than describe a single specific chromosome alteration. Figure 1 provides pictorial documentation of the band regions along chromosomes $1,3,6,7,9,10,11$ and 19 most frequently altered in this disorder.

Chromosome 1 alterations are without question the most frequent site of chromosome change in metastatic melanoma with up to $80 \%$ of tumors displaying numeric or structural alterations (particularly flanking the centromere). Nevertheless, chromosome 1 is the single most frequently altered chromosome in virtually every solid tumor, so the biologic significance (and tumor specificity) of these changes is currently indeterminate. Of interest, Nowell and colleagues [18] have described a nonreciprocal translocation involving chromosomes 1 and 19 which appears to describe a subset of patients with this disease $[\mathrm{t}(1 ; 19)(\mathrm{q} 12 ; \mathrm{p} 13)]$. The region involved in chromosome 1 is an area of constitutive heterochromatin (thus with little if any genic activity) while the region of chromosome 19 involved in this translocation apparently lies close to (but not involving) the chromosomal loci of the insulin receptor gene (INSR) [18].

Chromosome 6 (both the long and short arm) is also very frequently altered in metastatic melanoma, with the most common alteration a simple deletion of the long arm (6q-). The region which appears to be most consistently lost surrounds bands $6 \mathrm{q} 21$. In addition to chromosome 6 deletions, the involvement of chromosome 6 in a series of nonreciprocal translocations (primarily with chromosome 1 - see Table 1 ), is also a relatively frequent finding in this disorder [20]. This translocation $[t(1 ; 6)(q 11-12 ; q 15-21)]$, has been observed in both established melanoma cell lines and direct cultures, and interestingly the region on chromosome 1 involved in this translocation is identical to that involved in a translocation with chromosome 19 [18]. Although the site of the translocation on chromosome $6(q 12-13)$ is removed from that region most frequently involved in simple deletions (6q21), we have suggested that these alterations may in fact may both be related [20]. Specifically, the $t(1 ; 6)$ is a nonreciprocal translocation which in fact leads to the loss of sequences distal to $6 \mathrm{q} 11$. Thus, simple deletions as well as this nonreciprocal translocation both effectively remove sequences on 6q. Southern blotting using a panel of polymorphic DNA probes for chromoso-

Table 1. List of chromosome aberrations in malignant melanoma*

\begin{tabular}{|c|c|c|c|}
\hline $\begin{array}{l}\text { Region or } \\
\text { band }\end{array}$ & Type of aberration & Status* & References \\
\hline $1 \mathrm{p} 22-\mathrm{p} 11$ & $\begin{array}{l}\text { Deletions, } \\
\text { translocations }\end{array}$ & I & $\begin{array}{l}31 \\
12 \\
15 \\
16 \\
18\end{array}$ \\
\hline $1 q 11-q 12$ & $\begin{array}{l}\text { Translocations, } \\
\text { duplications }\end{array}$ & I & $\begin{array}{l}32 \\
12 \\
16 \\
18\end{array}$ \\
\hline $1 \mathrm{q} 11-\mathrm{q} 12$ & $t(1 ; 6)(q 11-12 ; q 15-21)$ & 1 & $\begin{array}{l}30 \\
20\end{array}$ \\
\hline $6 \mathrm{p} 11-\mathrm{q} 11$ & $\mathrm{i}(6 \mathrm{p})$, translocations & II & $\begin{array}{l}31 \\
12 \\
15\end{array}$ \\
\hline $6 \mathrm{q} 11-\mathrm{q} 27$ & $\begin{array}{l}\text { Deletions, } \\
\text { translocations }\end{array}$ & I & $\begin{array}{r}13 \\
12 \\
7 \\
15 \\
17 \\
18 \\
16\end{array}$ \\
\hline $6 \mathrm{q} 15-q 23$ & $t(1 ; 6)(q 11-12 ; q 15-21)$ & I & $\begin{array}{l}30 \\
20\end{array}$ \\
\hline $7 \mathrm{q} 11$ & Translocation & II & $\begin{array}{l}31 \\
12 \\
18 \\
16\end{array}$ \\
\hline
\end{tabular}

*From Mitelman I, Kaneko Y, and Trent J. Report on the Committee on Structural Chromosome Changes in Neoplasia (HGM10.5). Cytogenetic and Cell Genetics, 55: 358-386 (1990). + The criteria for each status as per HGM10.5 recommendations were: Status I - at least two laboratories have reported the abnormality in a total of five cases; Status II - at least three cases have been reported from two different laboratories. 
mal $6 \mathrm{q}$ has recently demonstrated a high percentage of loss of $6 \mathrm{q}$ alleles in the tumor cells of patients with metastatic melanoma [25]. The possible biologic relevance of this loss of chromosome $6 \mathrm{q}$ sequences in malignant melanoma will be described later in the text.

Additional chromosomes which have been shown to be altered in metastatic melanoma include chromosome 7 and 11. Chromosome 7 alterations most frequently involve the short arm (7p) and it is of interest that enhanced expression of the epidermal growth factor receptor (EGFR) gene (which maps to $7 \mathrm{p} 12$ ) is associated with an increased number of copies of chromosome $7 p$ in early stage melanomas [26]. Chromosome 11 abnormalities are more evenly divided between the short arm (particularly the terminal region) and the near distal long arm. Mitelman and colleagues [23] in a study of six cell lines from three melanoma patients have suggested that chromosome 11p alterations may play a role in melanoma tumorigenesis. The observation of $11 \mathrm{p}$ abnormalities in melanoma may relate somehow to the now well described presence of suppressor gene activity at 11p13 [27]. However, studies of malignant melanoma examining the loss of sequences on 11p using polymorphic probes has provided little evidence supporting the frequent loss of DNA within this chromosomal region $[25,28,29]$.

\section{Expression of silver stained nucleolar organizing regions (Ag-NORs) in human cancer}

In normal human cells the chromosomal regions associated with nucleolar formation (NORs) are present on the short arms of the acrocentric (Dand G-group of chromosomes) [33]. Simple histochemical techniques for selectively staining active NORs have been developed and can be used to cytologically evaluate the location and number of transcriptionally active NORs [34, 35]. Malignant tissue (in contrast to normal cells) in vivo often display cytologic irregularies in number, size and shape of nuclei. Accordingly, studies have appeared for several years comparing the cytologic expression of these ribosomal cistrons in tumor and normal tissue [36]. Studies of AgNORs in benign, dysplastic, and malignant melanocytic skin lesions have been performed by several laboratories [3740]. The results of Howat et al. [37] illustrate the general findings in melanoma for this procedure. These investigators applied the AgNOR technique to 30 benign nevi, 30 dysplastic nevi and 30 malignant melanomas. AgNORs in 200 nuclei were scored and the means calculated. A significant difference was shown to exist between the number of AgNORs per nucleus for malignant melanomas versus those for benign nevi or dysplastic nevi. Additionally, there was no statistically significant difference between benign and dysplastic nevi in terms of the total number of NORs per cell. The problem with this and related studies relates to a significant overlap in counts between benign and dysplastic tissue, leading to a limiting in the diagnostic potential of this procedure. Thus, the authors have suggested the AgNOR technique may prove to be of value in helping to differentiate malignant melanoma from dysplastic nevi, although it will be unlikely to be of help in separating dysplastic from benign nevi. While this method is still a research tool, it appears to be gaining some support as an adjunct to routine diagnostic histopathology.

\section{Relationship of cytogenetic abnormalities and clinical outcome in metastatic melanoma}

Although, the value of chromosomal analysis is well established in hematopoietic malignancies, the relationship between chromosome abnormalities and clinical outcome in solid tumors has received relatively little study. Recently a study was performed in order to determine the distribution of clonal chromosomal abnormalities in patients with metastatic melanoma, and to correlate these abnormal karyotypes with clinical information [41]. These very preliminary analyses revealed that selected cytogenetic abnormalities were in fact predictive of clinical outcome [41].

Sixty two patients were entered in this study with $46(74 \%)$ having sufficient metaphases for study. Ninety three percent of these patients had abnor- 


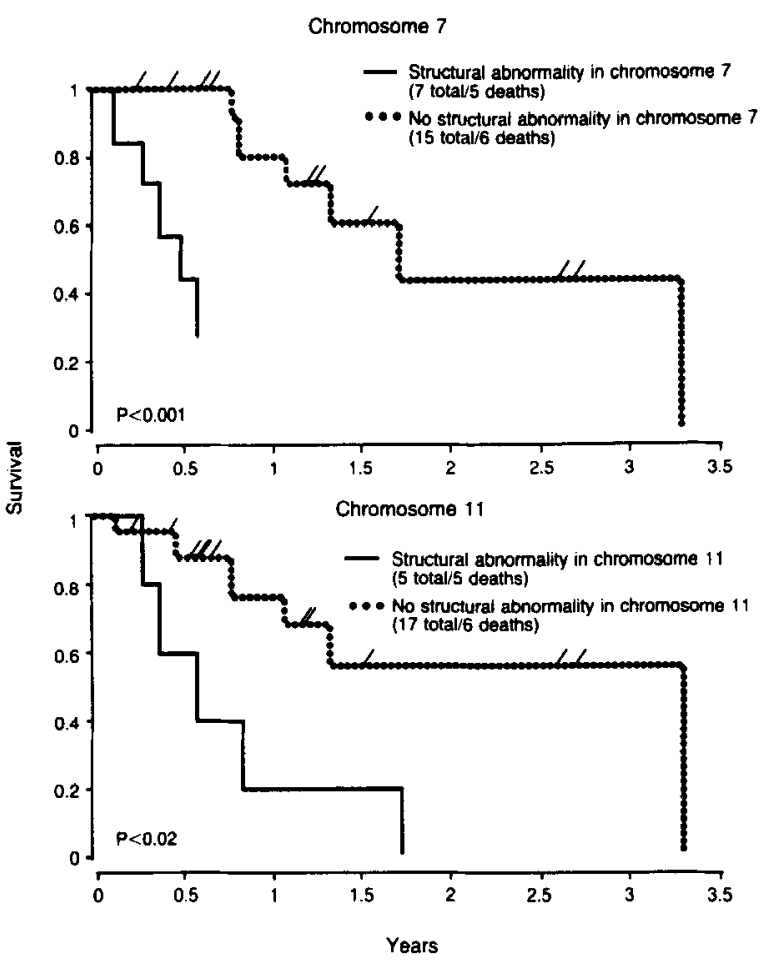

Fig. 2. Survival curves among patients who had stage III malignant melanoma with and without abnormalities in chromosomes 7 and 11. Diagonal lines intersecting survival curves denote patients for whom data were censored (used with permission)

mal karyotypes. There was no correlation between the presence or absence of normal mitoses and death rate $(\mathrm{P}=0.89)$.

We also examined the representativeness of our patient population, and the effect of considering particular subsets of patients in our analyses. Additionally results were examined for patients whose tissue samples were analyzed cytogenetically to determine whether survival differed from those patients for whom cytogenetic analysis could not be performed (e.g. no growth, no metaphases, etc.). The results of these studies indicated that none of the clinical or demographic features examined differed significantly [41].

When specific chromosome abnormalities were examined for their association with clinical outcome patients with a structural abnormality of chromosome 7 or 11 had a significantly shorter survival duration $(p<0.001$ and $p<0.02$, respectively) than patients without such chromosome ab- normalities (Fig. 2). This was true when survival was analyzed from the interval from cytogenetic study to death as well as the interval from diagnosis to death. Other karyotypic abnormalities examined (including those involving chromosomes 1, 3, 6,9 and 21) did not correlate significantly with a shorter survival period.

Finally, although the numbers involved in our study were clearly insufficient to support a definitive multivariant analysis, we did perform analyses of the relation of structural abnormalities of chromosome 7 and 11 to survival from biopsy, diagnosis and the occurrence of stage 3 disease until death; with the variables including age, sex, nodal involvement, the presence of visceral metastases, and stage 3 disease in a proportional-hazards model with an underlying Weibull survival distribution. The results of the univariant analysis (with regard to the relation of these structural abnormalities) remained significant when subjected to multivariant analyses [41].

In summary, this aforementioned report on karyotypic analyses on patients with metastatic melanoma examined the relation between banded chromosomal analyses and clinical outcome of disease for this important neoplasm. The results indicated that shortened survival duration was associated with patients with structural abnormalities of chromosome 7 and 11. Although the abnormalities involved in these chromosomes were diverse, the region of each chromosome was surprisingly consistent. For example the rearrangements of chromosome 7 almost exclusively involved the short arm (25/27 cases). Similarly there was a clustering of chromosomal breakpoints along chromosome 11 , which were about equally divided between the short arm (p11-p14) and the long arm (q23-q25). The underlying biology related to the observed difference in survival is clearly not understood. Several cellular oncogenes map to chromosome 7 and 11 but the influence of these oncogenes (if any) on the negative prognostic effects of alterations of chromosome 7 or 11 is unclear.

Finally, because of the large number of comparisons which were necessitated by the study design and the number of individual chromosomes examined, these results clearly require corroboration. 
Nevertheless, in as much as cytogenetic analysis is providing useful information about hematopoietic malignancies, it appears likely that analysis of solid tumors (such as melanoma) will prove to be of similar value.

\section{Biologic relevance of chromosome abnormalities in malignant melanoma}

As mentioned previously molecular examination of specific chromosomal abnormalities has pinpointed the site of genes important in the process of growth regulation. Because malignant transformation is envisioned as a multistep process [42], recent studies highlighting the importance of tumor suppressor genes in the development of several human cancers are particularly of interest [43]. The supporting evidence for tumor suppressor genes is based upon a variety of studies including: 1) cytogenetic evidence for deletion of specific chromosomal regions, 2) loss of heterozygosity examined by RFLP analysis, and 3) study of somatic cell fusion experiments. The following section will briefly document an experimental approach using somatic cell fusion to assign a potentially important gene for malignant melanoma to human chromosome 6 .

As mentioned previously chromosome banding analysis of human malignant melanoma has documented the nonrandom alteration of chromosome 6 (particularly the deletion of the long arm). In order to determine the relevance of chromosome 6 abnormalities in melanoma, a normal chromosome 6 was directly introduced into melanoma cell lines [44]. In these studies a human-mouse microcell hybrid was generated that contained a single copy of chromosome 6 (derived from a normal human diploid fibroblast) as its only human component.

Table $2^{a}$. Characteristics of the parental and microcell line

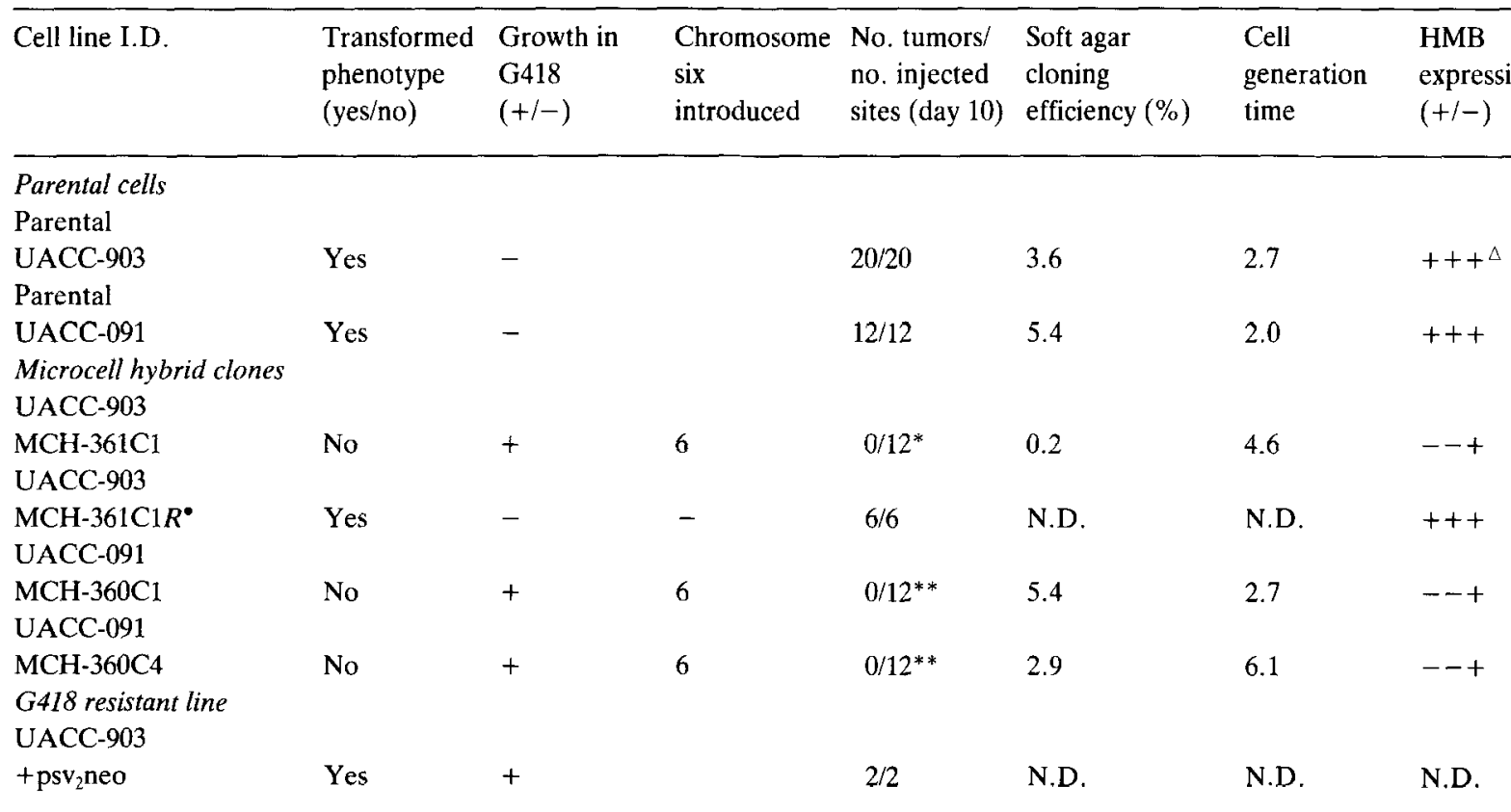

\footnotetext{
$\triangle$ Reactivity to HMB-45 was quantitated as uniformly positive $[>80 \%$ of all tumor cells postive $(+++)]$ to focal reactivity $[<10 \%$ of tumor cells positive $(--+)$ ] see Fig. 2.

- This revertant line was produced by culturing cells from a tumor formed following injection of $\mathrm{MCH}-361 \mathrm{C} 1$.

* Tumors arose in all animals after growth delay of up to 34 days. In every case examined, loss of the introduced 6 [assessed by cytogenetic and RFLP analysis (e.g. Fig. 3B)] occurred coincident with tumor formation.

** Following 60 days, $40 \%$ of animals developed tumors. However, loss of the introduced 6 was again confirmed in all cases by directly analyzing tumor tissue by RFLP analysis.

${ }^{a}$ Used with permission.
} 


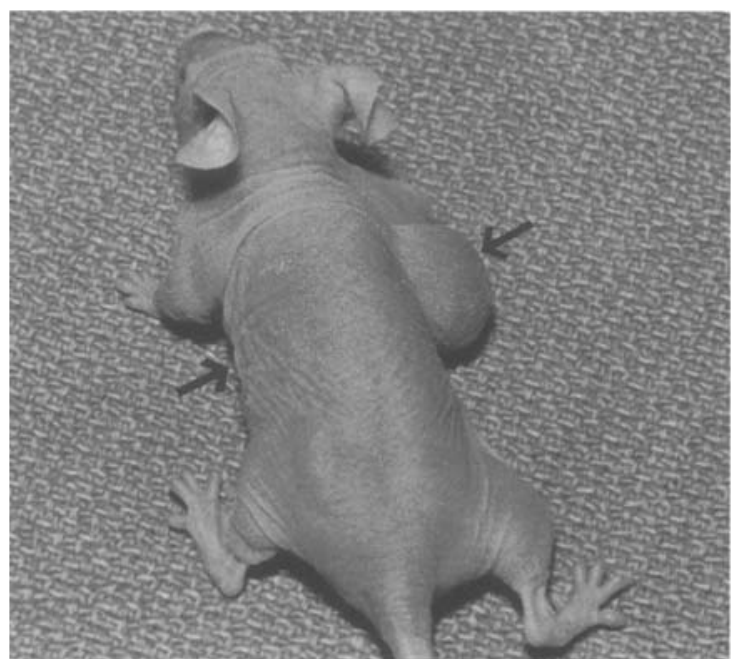

Fig. 3. Tumor formation 9 weeks following injection of $1 \times 10^{7}$ cells from a malignant melanoma cell line into the right flank of a nude (nu/nu) mouse. The identical number of cells from the $(+6)$ microcell hybrid of this same cell line were injected at the same time into the left flank of this animal but were suppressed for tumor formation (see ref. 44).

The retention of chromosome 6 resulted from the insertion of the neomycin antibiotic resistance gene ( $\mathrm{psv}_{2} \mathrm{neo}$ ) into this chromosome 6, and subsequent selection of microcell hybrids with medium containing the neomycin analog G418. This hybrid was then used to transfer an entire normal chromosome 6 into two different human melanoma cell lines via microcell transfer. A summary of characteristics of the parental and microcell line is presented in Table 2 .

To summarize these results, the in vitro morphology of one of the two hybrid lines changed dramatically in response to the introduction of human chromosome 6 . Both cell lines showed a diminution of melanoma associated antigen expression on $(+6)$ microcell hybrids, one of the two microcell hybrids showed a reduction in soft agar clonogenicity, and both showed a lengthening of cell generation time. With this significant decline in melanoma associated antigen expression (together with the finding of altered cell morphology and diminished agar clonogenecity of $(+6)$ microcell hybrids), a study was undertaken to determine whether the introduction of normal chromosome 6 would lead to suppression of tumor growth in nude mice.

After injection of cells $\left(5 \times 10^{6}-1 \times 10^{7}\right)$ from the parental cell lines and $(+6)$ microcell hybrids into athymic (nu/nu) mice. Rapid growth occurred in $100 \%$ of animals from the parental lines within 5 to 7 days (Table 2, Fig. 3). For both tumor lines tested tumor formation was suppressed in animals receiving the $(+6)$ when compared to the parental line. However, in several animals tumors developed following initial suppression, and the outgrowth from these $(+6)$ microcell 'revertants' were subsequently shown to have lost the introduced chromosome 6 [44]. As a control for the introduction of chromosome 6 into the melanoma cell lines the $\mathrm{psv}_{2}$ neo gene was inserted by electroporation into melanoma cell lines and in contrast to the introduction of the chromosome 6 , the addition of the selectable marker did not alter in vitro morphology or affect tumorigenicity.

These results have suggested that it may be reasonable to speculate that genetic information present on chromosome 6 can suppress the malignant phenotype of human melanoma cells. Following the suggestion of Vogelstein [45], tumor suppressor genes may have a hierarchial and incremental affect on the regulation of cell growth and our results may therefore suggest that this gene(s) on chromosome 6 may be acting early in the pathway of tumor formation.

Finally, because chromosome 1 is the most frequently altered chromosome in malignant melanoma, it was of significant interest when Bale et al. [46] recently mapped a gene for hereditary cutaneous malignant melanoma-dysplastic nevus to chromosome 1p using molecular genetic techniques and multipoint linkage. The authors speculated that this discovery could lead to a starting point for identifying a gene(s) for this disorder and eventually lead to a characterization of its function. However, the general significance of this finding is still unclear as other groups have failed to demonstrate linkage on $1 \mathrm{p}$ for families with this disorder, and further, studies from this same group of loss of alleles from the distal part of chromosome 1 appear to occur late (rather than early) in melanoma tu- 
mor progression [47]. Nevertheless, this work is indeed of interest as a genetic component to the hereditary forms of this disease must certainly exist, and sequences on 1p may to play a role in the etiology of this disorder.

In summary then, on cytogenetic grounds the most highlighted karyotypic abnormalities in early melanomas involve chromosomes 9 and 10, while in metastatic melanoma these chromosomes are infrequently altered and instead there is the frequent involvement particularly of chromosomes 1 and 6. Further, the only chromosomal loci implicated in hereditary cutaneous malignant melanoma is chromosome $1 \mathrm{p}$, but study of melanoma tumors appears to indicate that loss of sequences in this region are in fact late rather than early events in the development of this cancer. The conclusion then to be drawn is that much work remains to be done in order to understand the molecular basis for this important disorder. We should be prepared for surprises!

\section{Conclusions}

This review has provided a brief update on current progress in identifying recurring sites of chromosome change in human malignant melanoma. It is clear that additional work is required to more completely characterize this disorder, nevertheless several inferences can be made.

- Early stage melanomas and premelanotic lesions are characterized by minimal karyotypic alterations and the possible alteration of chromsomes $9 \mathrm{p}$ and $10 \mathrm{q}$.

- Examination of metastatic melanoma implicates a subset of chromosomes in this disorder (particularly 1, 6, 7 and 11).

- Analysis of AgNORs in melanoma may provide a useful adjunct to routine histopathology in distinguishing overt melanotic lesions from dysplastic or benign nevi.

- Clinical correlations of chromosome change in malignant melanoma are very preliminary. By analogy to work in hematologic malignancies these studies may in the future provide impor- tant insights into chromosomal regions whose alteration relates to clinical outcome.

- Biologic implications of chromosomal abnormalities in melanoma are also in their infancy. However, evidence for suppressor gene activity on chromosome 6 has been obtained.

- Continued study of the basic genetics of malignant melanoma should add significantly to our understanding of the causation and progression of this disorder.

\section{Acknowledgements}

Research supported in part by CA29476 and CA41183 awarded by the National Cancer Institute/National Institutes of Health, Bethesda, MD.

\section{References}

1. Lee JAH: Etiology, epidemiology, and prognosis of melanoma and skin neoplasms. Current Opinion in Onc 2: 388393, 1990

2. Mitelman F: Catalog of chromosome aberrations in cancer, 4th Edition. New York, Alan R. Liss (in press)

3. Mitelman F, Kaneko Y, Trent JM: Report of the committee on chromosome changes in neoplasia. Cytogenet and Cell Genet 55: 358-386, 1990

4. Jaspers NGJ, Roza-de Jongh EJM, Donselaar IG, Van Velzen-Tillemans JTM, van Hemel JO, Rumke P, van der Kamp AWM: Sister chromatid exchanges, hyperdiploidy and chromosomal rearrangements studied in cells from melanoma-prone individuals belonging to families with the dysplastic nevus syndrome. Cancer Genet Cytogenet 24: 33-43, 1987

5. Caporaso N, Greene MH, Tsai S, Pickle LW, Mulvihill JJ: Cytogenetics in hereditary malignant melanoma and dysplastic nevus syndrome: Is dysplastic nevus syndrome a chromosome instability disorder? Cancer Genet Cytogenet 24: 299-314, 1987

6. Lynch HT, Fusaro RM, Danes BS, Kimberling WJ, Lynch JF: A review of hereditary malignant melanoma including biomarkers in familial atypical multiple mole melanoma syndrome. Cancer Genet Cytogenet 8: 325-358, 1983

7. Sanford KK. Tarone RE, Parshad R, Tucker MA, Greene MH, Jones $G M$ : Hypersensitivity to $G_{2}$ chromatid radiation damage in famillial dysplastic naevus syndrome. Lancet ii: $1111-1116,1987$

8. Cowan JM, Halaban R, Francke U: Cytogenetic anlysis of 
melanocytes from premalignant nevi and melanomas. J Natl Cancer Inst 80: 1159-1164, 1988

9. Parmiter AH, Balaban G, Clark Jr WH, Nowell PC: Possible involvement of the chromosome region 10q24-> q26 in early stages of melanocytic neoplasia. Cancer Genet Cytogenet 30: 313-317, 1988

10. McDonagh AJG, Wright AL, Messenger AG: Dysplastic naevi in association with partial deletion of chromosome 11. Clinical and Exper Derm 15: 44-45, 1990

11. Trent J, Olson S, Lawn R: Chromosomal localization of human leukocyte, fibroblast and immune interferon genes by means of in situ hybridization. PNAS 79: 7809, 1982

12. Becher R, Gibas Z, Karakousis C, Sandberg AA: Nonrandom chromosome changes in malignant melanoma. Cancer Res 43: 5010-5016, 1983

13. Trent JM, Rosenfeld SB, Meyskens FL: Chromosome 6q involvement in human malignant melanoma. Cancer Genet Cytogenet 9: 177-180, 1983

14. Pathak S, Drwinga HL, Hsu TC: Involvement of chromosome 6 rearrangements in human malignant melanoma cell lines. Cytogenet Cell Genet 36: 573-579, 1983

15. Balaban G, Herlyn M, Guerry D, Bartolo R, Koprowski H, Clark WH, Nowell PC: Cytogenetics of human malignant melanoma and premalignant lesions. Cancer Genet Cytogenet 11: 429-439, 1984

16. Pedersen MI, Bennett JW, Wang N: Nonrandom chromosome structural aberrations and oncogene loci in human malignant melanoma. Cancer Genet Cytogenet 20: 11-27, 1986

17. Cowan JM, Halaban R, Lane AT, Francke U: The involvement of $6 \mathrm{p}$ in melanoma. Cancer Genet Cytogenet 20: 255, 1986

18. Parmiter AH, Balaban G, Herlyn M, Clark WH, Nowell PC: A $\mathrm{t}(1 ; 19)$ chromosome translocation in three cases of human malignant melanoma. Cancer Res 46: 1526-1529, 1986

19. Trent JM, Thompson FH, Meyskens FL: Cytogenetics of melanoma. In: T Slaga (ed.) Skin Cancer Biology. Raven Press, New York, pp 165-186, 1989

20. Trent JM, Thompson FH, Meyskens Jr FL: Identification of a recurring translocation site involving chromosome 6 in human malignant melanoma. Cancer Res 49: 420-423, 1989

21. Grammatico P, Lo Re ML, Scarpa S, Modesti A, Del Porto G: Human malignant melanoma. Significance of chromosomal abnormalities. Cancer Genet Cytogenet 48: 237-242, 1990

22. Limon J, Dal Cin P, Sait SNJ, Karakousis C, Sandberg AA: Chromosome changes in metastatic human melanoma. Cancer Genet Cytogenet 30: 201-211, 1988

23. Heim S, Mandahl N, Arheden K, Giovannella BC, Yim SO, Stehlin Jr JS, Mitelman F: Multiple karyotypic abnormalities, including structural rearrangements of $11 \mathrm{p}$, in cell lines from malignant melanomas. Cancer Genet Cytogenet 35: 5-20, 1988

24. Kacker RK, Giovanella BC, Pathak S: Consistent karyo- typic abnormalities in human malignant melanomas. Anticancer Res 10: 859-872, 1990

25. Millikin D, Meese E, Witowski C, Meltzer P, Trent JM: Loss of constitutional heterozygosity for loci on chromosome $6 \mathrm{q}$ in human malignant melanoma. Cancer Res, in press, 1991

26. Koprowski H, Herlyn M, Balaban G, Parmiter A, Ross A, Nowell P: Expression of the receptor for epidermal growth factor correlates with increased dosage of chromosome 7 in malignant melanoma. Somatic Cell and Mol Genet 11: $297-$ 302, 1985

27. Fearon ER, Vogelstein B, Feinberg AP: Somatic deletion and duplication of genes on chromosome 11 in Wilms' tumours. Nature 309: 176-178, 1984b

28. Dracopoli NC, Houghton AN, Old LJ: Loss of polymorphic restriction fragments in malignant melanoma: implications for tumor heterogeneity. Proc Natl Acad Sci 82: 14701474,1985

29. Nordenskjold M, Werelius B, Ringborg U: Allele losses in malignant melanoma reflect random events. Anticancer Res 10: 883-886, 1990

30. Muir PD, Gunz FW: A cytogenetic study of eight human melanoma cell cell lines. Pathology 11: 597-606, 1979

31. Kakati S, Song SY, Sandberg AA: Chromosomes and causation of human cancer and leukemia. XXII, Karyotypic changes in malignant melanoma. Cancer 40: 1173-1181, 1977

32. Atkin NB, Baker MC: A metastatic malignant melanoma with 24 chromocomes. Hum Genet 58: 217-219, 1981a

33. Ferguson-Smith MA: The sites of nucleolus formation in human pachytene chromosomes. Cytogenet 3: 124-134, 1964

34. Goodpasture C, Bloom SE: Visualization of nucleolar organizing regions in mammalian chromosomes using silver stain. Chromosoma 53: 37-50, 1975

35. Howell WM, Denton TE, Diamond JR: Differential staining of human acrocentric chromosomes. Experientia 31: 260-262, 1975

36. Trent JM, Carlin DA, Davis JR: Expression of silverstained nucleolar organizing regions (Ag-NORs) in human cancer. Cytogenet Cell Genet 30: 31-38, 1981

37. Howat AJ, Wright AL, Cotton DWK, Reeve S, Bleehen SS: AgNORs in benign, dysplastic, and malignant melanocytic skin lesions. American Jr Derm 12(2: 156-161, 1990

38. Crocker J, Skibeck N: Nucleolar organiser region-associated proteins in cutaneous melanotic lesions: a quantitative study. J Clin Pathol 40: 885-889, 1987

39. Howat AJ, Giri DD, Cotton DWK, Slater DN: Nucleolar organiser regions in Spitz nevi and malignant melanomas. Cancer 63: 474-478, 1989

40. Egan M, Crocker J: Nucleolar organiser regions in cutaneous tumours. J Pathol 154: 247-253, 1988

41. Trent JM, Meyskens FL, Salmon SI, Ryschon K, Leong SPL, Davis JR, McGee DL: Relation of cytogenetic abnormalities and clinical outcome in metastatic melanoma. N Engl J Med 322: 1508-1511, 1990 
42. Knudson Jr AG, Hethcote HW, Brown BW: Mutation of childhood cancer: A probabilistic model for the incidence of retinoblastoma. Proc Natl Acad Sci USA 72: 5116-5120, 1975

43. Friend SH, Drya TP, Weinberg RA: Oncogenes and tumor-suppressing genes. N Engl J Med 318: 618, 1988

44. Trent JM, Standbridge EJ, McBride HL, Meese EU, Casey G, Araujo DE, Witkowski CM, Nagle RB: Tumorigenicity in human melanoma cell lines controlled by introduction of human chromosome 6. Science 247: 568-571, 1990

45. Vogelstein B, Fearon ER, Kern SE, Hamilton SR, Preisinger AC, Nakamura Y, White R: Allelotype of colorectal carcinomas. Science 244: 207-211, 1989

46. Bale SJ, Dracopoli NC, Tucker MA, Clark Jr WH, Fraser MC, Stanger BZ, Green P, Donis-Keller H, Housman DE,
Greene MH: Mapping the gene for hereditary cutaneous malignant melanoma-dysplastic nevus to chromosome 1p. N Engl J Med 320: 1367-1372, 1989

47. Dracopoli NC, Harnett Bale SJ et al.: Loss of alleles from distal chromosome lp occurs late in melanoma tumor progression. Proc Natl Acad Sci USA 86: 4614-4618, 1989

Address for offprints:

Jeffrey M. Trent,

Emanuel N. Maisel Professor Of Oncology,

Department of Radiation Oncology and Human Genetics, 1150 W. Medical Center Drive,

University of Michigan,

An Arbor, MI 48109-0668, USA 